www.nature.com/clinicalpractice/uro

\title{
Why should we increase public awareness of bladder cancer, and how can we do it?
}

\author{
Edward M Messing
}

Bladder cancer is the fifth most commonly diagnosed noncutaneous malignancy in the US, and the second most prevalent among middleaged and elderly men. Methods of detection, prevention and treatment have hardly changed over the past 20 years, as, consequently, has the likelihood of dying from this disease; new approaches are desperately needed.

Increased basic, translational, clinical and health services research will be needed to devise strategies that can be brought to clinical fruition, which will in turn depend on attracting the best scientists into bladder cancer research. The NIH has, however, cut research support for bladder cancer by $7 \%$, while it increased funding for the other ten most commonly diagnosed malignancies by 4-100\% between 2002 and 2005. In 2005, bladder cancer research received the lowest funding per new case, one of the lowest per cancer-associated death, and was the subject of the fewest clinical trials of all the common cancers. Ironically, a huge amount is still spent on bladder cancer because it is the most expensive malignancy to treat per patient over their lifetime. An ounce of prevention (or research on prevention) would, therefore, certainly be worth a pound of cure.

What might change matters? I believe that increased support for research from organized patient advocacy groups, and the application of political and economic pressure/influence to stimulate governmental and foundation funding, would be an excellent start. Advocacy has prompted research for other urologic diseases, such as prostate cancer and interstitial cystitis. The importance of basic research in developing new molecular therapeutic targets has been borne out in another common urologic malignancy, renal cell carcinoma. Advocacy, of course, requires dedicated champions, often patients and their families, generous patrons
A little

additional

time spent

to increase

awareness

has the

potential for

an enormous

payback

EM Messing is the WW Scott Professor

of Urology, the Chair of the Department of Urology, and a Professor of Oncology and Pathology at the James P Wilmot Cancer Center, University of Rochester School of Medicine, NY, USA.

Competing interests

The author declared no competing interests.

www.nature.com/clinicalpractice doi:10.1038/ncpuro1061 and 'poster boys', to act as spokespeople. In the US, a major, national bladder cancer support group, the Bladder Cancer Advocacy Network (BCAN), has been developed by its truly dedicated and tireless founders Diane and John Quale (www.bcan.org); however, political advocates, philanthropists and poster boys have yet to step forward.

What can be done? Urologists and other health-care workers who treat patients with bladder cancer can increase awareness and advocacy. Reporting the statistics mentioned above to the families of all our patients with bladder cancer should stimulate grass roots support, and directing this support to organizations, such as the BCAN and other advocacy groups, might focus efforts tremendously.

Three additional issues should be highlighted in any awareness campaign. Firstly, there is a direct causal relationship between cigarette smoking and the occurrence of bladder cancer, and between continued smoking and disease progression. Secondly, the public and our colleagues in primary care need to be made aware of the highly intermittent nature of bladder-cancer-induced hematuria to enable earlier diagnosis. Finally, corporate interests and regulatory bodies must be reminded that bladder cancer is not only a common and serious cancer, but also a chemosensitive one, which is ideal for the use of various novel endoscopic and radiographic imaging approaches. This information should stimulate investment in technological and pharmacological advances, which could help improve clinical outcomes.

It is time for all urologists who treat this disease to think beyond the care of individual patients. A little additional time spent to increase awareness has the potential for an enormous payback. 